\title{
Pressure-pulse interaction with the magnetosphere and ionosphere
}

\author{
D. G. Sibeck, ${ }^{1}$ N. B. Trivedi, ${ }^{2}$ E. Zesta, ${ }^{3}$ R. B. Decker, ${ }^{4}$ H. J. Singer, ${ }^{5}$ A. Szabo, ${ }^{1}$ \\ H. Tachihara, ${ }^{6}$ and J. Watermann ${ }^{7}$ \\ Received 8 September 2002; revised 18 October 2002; accepted 3 December 2002; published 28 February 2003.
}

[1] We reexamine traveling convection vortices (TCVs) seen by the Magnetometer Array for Cusp and Cleft Studies on 9 November 1993. IMP-8 energetic ion observations confirm that the solar wind pressure variations previously associated with these TCVs were generated by kinetic processes within the Earth's foreshock. As expected during this interval of spiral IMF orientation, fast mode waves launched by the pressure variations first arrived in the equatorial ionosphere near dusk and propagated dawnward. We derive a model for the field-aligned currents generated by transient compressions of the magnetopause and show that it accounts for the number of TCVs seen in the prenoon ionosphere, their sense of rotation, the latitude at which they occur, and their absence in the postnoon ionosphere. INDEX TERMS: 2784 Magnetospheric Physics: Solar wind/magnetosphere interactions; 2154 Interplanetary Physics: Planetary bow shocks; 2724 Magnetospheric Physics:

Magnetopause, cusp, and boundary layers; 2431 Ionosphere: Ionosphere/magnetosphere interactions (2736); KEYWORDS: traveling convection vortices, pressure pulses

Citation: Sibeck, D. G., N. B. Trivedi, E. Zesta, R. B. Decker, H. J. Singer, A. Szabo, H. Tachihara, and J. Watermann, Pressurepulse interaction with the magnetosphere and ionosphere, J. Geophys. Res., 108(A2), 1095, doi:10.1029/2002JA009675, 2003.

\section{Introduction}

[2] Transient events with durations ranging from 5 to 20 min are common in high-latitude dayside ground magnetograms. An unknown fraction of the events can be interpreted in terms of the propagating swirls of ionospheric convection known as traveling convection vortices (TCVs) [Friis-Christensen et al., 1988]. Because events reach peak amplitudes $(\sim 100 \mathrm{nT})$ at the high-latitude feet of magnetic field lines that map to the vicinity of the dayside magnetopause, they are generally attributed to unsteady solar windmagnetosphere interaction processes like bursty merging [Lanzerotti et al., 1986], the Kelvin-Helmholtz instability [McHenry et al., 1988], or pressure-pulse driven boundary waves on the magnetopause [Sibeck et al., 1989].

[3] A number of observations favor the pressure-pulse interpretation. The events generally correspond to sudden impulses (SI) in lower latitude ground magnetograms and at geosynchronous orbit [Sibeck, 1993; Trivedi et al., 2002]. They move through high-latitude ground magnetometer chains and geosynchronous orbit in the direction predicted for interplanetary magnetic field (IMF) discontinuities

\footnotetext{
${ }^{1}$ NASA Goddard Space Flight Center, Greenbelt, Maryland, USA.

${ }^{2}$ Laboratório de Ciências Espaciais de Santa Maria, Santa Maria, RS, Brazil.

${ }^{3}$ Atmospheric Sciences, University of California, Los Angeles, Los Angeles, California, USA.

${ }^{4}$ Johns Hopkins University/Applied Physics Laboratory, Laurel, Maryland, USA.

${ }^{5}$ NOAA Space Environment Center, Boulder, Colorado, USA.

${ }^{6}$ Earth and Planetary Sciences, University of Kyushu, Fukuoka, Japan.

${ }^{7}$ Danish Meteorological Institute, Copenhagen, Denmark.
}

sweeping across the magnetosphere [Korotova et al., 1999, 2002]. Efforts to determine whether the same SI signatures also propagate through the equatorial ionosphere in the direction predicted for solar wind features striking the magnetopause have been hampered by the absence of hightime resolution digital ground magnetograms. Statistical surveys argue against interpretations in terms of bursty merging or the Kelvin-Helmholtz instability [Sibeck and Korotova, 1996]; the high-latitude events do not occur preferentially for either southward IMF orientations or enhanced solar wind velocities.

[4] A major problem impedes interpretation of the events in terms of solar wind pressure variations striking the magnetosphere. Although the ground events occur during intervals of enhanced IMF variability, they do not occur during intervals of enhanced solar wind dynamic pressure variability [Konik et al., 1994]. In fact, abrupt variations in the solar wind dynamic pressure have only been identified for a few ground events [e.g., Friis-Christensen et al., 1988; Sibeck et al., 1989]. Perhaps the pressure variations that produce the events cannot be seen far upstream because they are generated in the foreshock near the bow shock [Sibeck and Korotova, 1996].

[5] Kivelson and Southwood [1991], Glaßmeier and Heppner [1992], and Lysak et al. [1994] have presented magnetohydrodynamic models that make conflicting predictions for event characteristics in the high-latitude ionosphere. Whereas Kivelson and Southwood predicted that event amplitudes should peak on magnetic field lines that map to the abrupt density gradients at the inner edge of the low-latitude boundary layer (LLBL), Glaßmeier and Heppner predicted that event amplitudes should peak on magnetic field lines that map to the magnetopause. In the model reported by Lysak et al., the events form at locations where 
Table 1. Locations of Ground Magnetometers

\begin{tabular}{llrrr}
\hline & \multicolumn{1}{c}{ Name } & CGLAT & CGLONG & Local Noon (UT) \\
\hline ALC & Alcantra & 0.7 & 29.3 & 1500 \\
AMK & Tasiilaq & 69.3 & 54.6 & 1315 \\
ANC & Ancon & 1.6 & 354.6 & 1706 \\
ARI & Ariquemes & 1.7 & 8.4 & 1612 \\
BLM & Belem & 1.7 & 25.6 & 1500 \\
CD & Cape Dorset & 74.6 & 1.2 & 1645 \\
CH & Coral Harbour & 74.8 & 349.2 & 1730 \\
DMH & Danmarkshavn & 77.2 & 87.1 & 1038 \\
FHB & Paamiut & 68.0 & 39.7 & 1426 \\
GH & Gjoa Haven & 78.2 & 323.4 & 1915 \\
GUA & Guam & 5.6 & 215.6 & 0220 \\
IG & Igloolik & 79.4 & 351.5 & 1720 \\
MCE & Magic-1 East & 75.5 & 66.4 & 1220 \\
MOK & Mokolo & 1.9 & 85.1 & 1106 \\
NRD & Nord & 80.9 & 105.8 & 0917 \\
PB & Pelly Bay & 78.6 & 335.5 & 1825 \\
PG & Pangnirtung & 75.2 & 20.1 & 1500 \\
PRD & Peradania & -0.1 & 152.3 & 0636 \\
RB & Repulse Bay & 76.9 & 343.2 & 1755 \\
SCO & Ittoqqortoormiit & 71.6 & 73.2 & 1143 \\
SKT & Maniitsoq & 72.0 & 38.0 & 1434 \\
SLZ & Saõ Luis & 0.5 & 29.6 & 1500 \\
SMA & Santa Maria & -19.3 & 13.2 & 1500 \\
TER & Teresina & -1.0 & 29.7 & 1500 \\
UPN & Upernavik & 79.5 & 42.0 & 1417 \\
\hline & & & &
\end{tabular}

field-line resonances occur. Also, whereas the Glaßmeier/ Heppner model predicts that a transient compression of the magnetosphere should generate a pair of oppositely directed field-aligned currents and vortices, both the Kivelson/ Southwood and Lysak et al. models predict that it will generate a triplet. All the models concur on one point; the ionospheric events should only be present at local times where the transmitted solar wind variations apply azimuthal pressure gradients to the magnetosphere.

[6] As the above discussion indicates, a number of questions concerning the origin and characteristics of transient events in high-latitude ground magnetograms remain unanswered. This paper compares previously reported observations of TCVs on 9 November 1993 with predictions of first-principle models and simulations. We begin by comparing IMP-8 solar wind plasma, magnetic field, and energetic ion observations with the predictions of hybrid code simulations to demonstrate that the solar wind dynamic pressure pulses observed on this day were generated within the foreshock. We then derive a relationship for the field-aligned currents generated when pressure variations are applied to the magnetosphere. We reconcile the predictions of this model with the observations on 9 November 1993 and comment on the conflicting predictions of the Kivelson/Southwood, Glaßmeier/Heppner, and Lysak et al. models. Finally, we use high-time resolution observations to demonstrate that the motion of the corresponding SI signatures through the equatorial ionosphere was consistent with that predicted for fast mode waves launched into the magnetosphere by pressure variations aligned with the IMF.

\section{Data Sets}

[7] To determine the origin of the pressure variations seen upstream from the bow shock and the nature of their interaction with the magnetosphere, we will present IMP8 solar wind observations by the GSFC magnetometer, MIT plasma, and JHU/APL EPE energetic particle instruments
[King, 1982]. The triaxial fluxgate magnetometer samples the vector magnetic field every $40 \mathrm{~ms}$ to provide averages at a maximum rate of once in every $0.325 \mathrm{~s}$. Plasma parameters from the Faraday cup detector were obtained from a nonlinear, least squares fit of the sum of the sector currents in each energy/charge window to those expected from a convecting, isotropic Maxwellian velocity distribution function. The data to compute the fits were taken within eight spins, i.e., $\sim 20.8 \mathrm{~s}$, once in every $58 \mathrm{~s}$. The F detector of the EPE instrument has a geometric factor of $0.0321 \mathrm{~cm}^{2} \mathrm{sr}$ and is canted to look $45^{\circ}$ above the ecliptic plane. At half efficiency, it measured ions with energies greater than 38 $\mathrm{keV}$ and electrons with energies greater than $22 \mathrm{keV}$ in one $2.64 \mathrm{~s}$ spin every $20.8 \mathrm{~s}$. Because energetic electrons are rare in foreshock events [Lin et al., 1974], the F detector essentially measures ions.

[8] To illustrate the motion of the events through geosynchronous orbit, we will present GOES-6 and -7 magnetic field observations at $3 \mathrm{~s}$ time resolution [Rufenach et al., 1992]. To time the westward motion of TCVs through North America, we will present Magnetometer Array for Cusp and Cleft Studies (MACCS) observations at $5 \mathrm{~s}$ time resolution [Hughes and Engebretson, 1997]. To demonstrate that no TCVs were observed postnoon, we will present Greenland [Wilhjelm and Friis-Christensen, 1976] ground magnetometer observations at $20 \mathrm{~s}$ time resolution. Finally, to track the motion of corresponding signatures through equatorial ground magnetograms, we will present Equatorial Magnetometer network [Tachihara et al., 1996] observations at $3 \mathrm{~s}$ time resolution. Table 1 lists the locations of the ground magnetometers used in this study, together with the times when they pass through magnetic local noon. Figure 1 presents their locations.

\section{Solar Wind Observations}

[9] Thomas and Brecht [1988] reported the results of hybrid code simulations for spatially limited low-density beams of suprathermal ions fired sunward (along the $z$ axis in their simulation) along IMF lines into the oncoming solar wind. The counterstreaming plasma populations triggered ion cyclotron waves, which then scattered both particle populations. The end result was a single hot ion population moving antisunward at bulk velocities slightly slower than those in the pristine solar wind. As the simulation results presented in Figure 2 indicate, the enhanced pressures associated with the hot ion population excavated a cavity of depressed densities and magnetic field strengths bounded by regions of enhanced densities and magnetic field strengths. The strength of these perturbations increased steadily with time.

[10] As noted by Thomas and Brecht [1988], the results of their simulation must be directly applicable to the foreshock, where a small fraction of the incident solar wind ions is reflected to form greatly energized, spatially limited, sunward-streaming beams. Both the waves in the magnetic field that couple the counterstreaming solar wind and reflected ion populations and the resulting diffuse ion populations typify the region upstream from the Earth's quasi-parallel bow shock [Greenstadt et al., 1970; Paschmann et al., 1981]. Rapid variations in the IMF orientation connect some bundles of IMF field lines to the bow shock, 


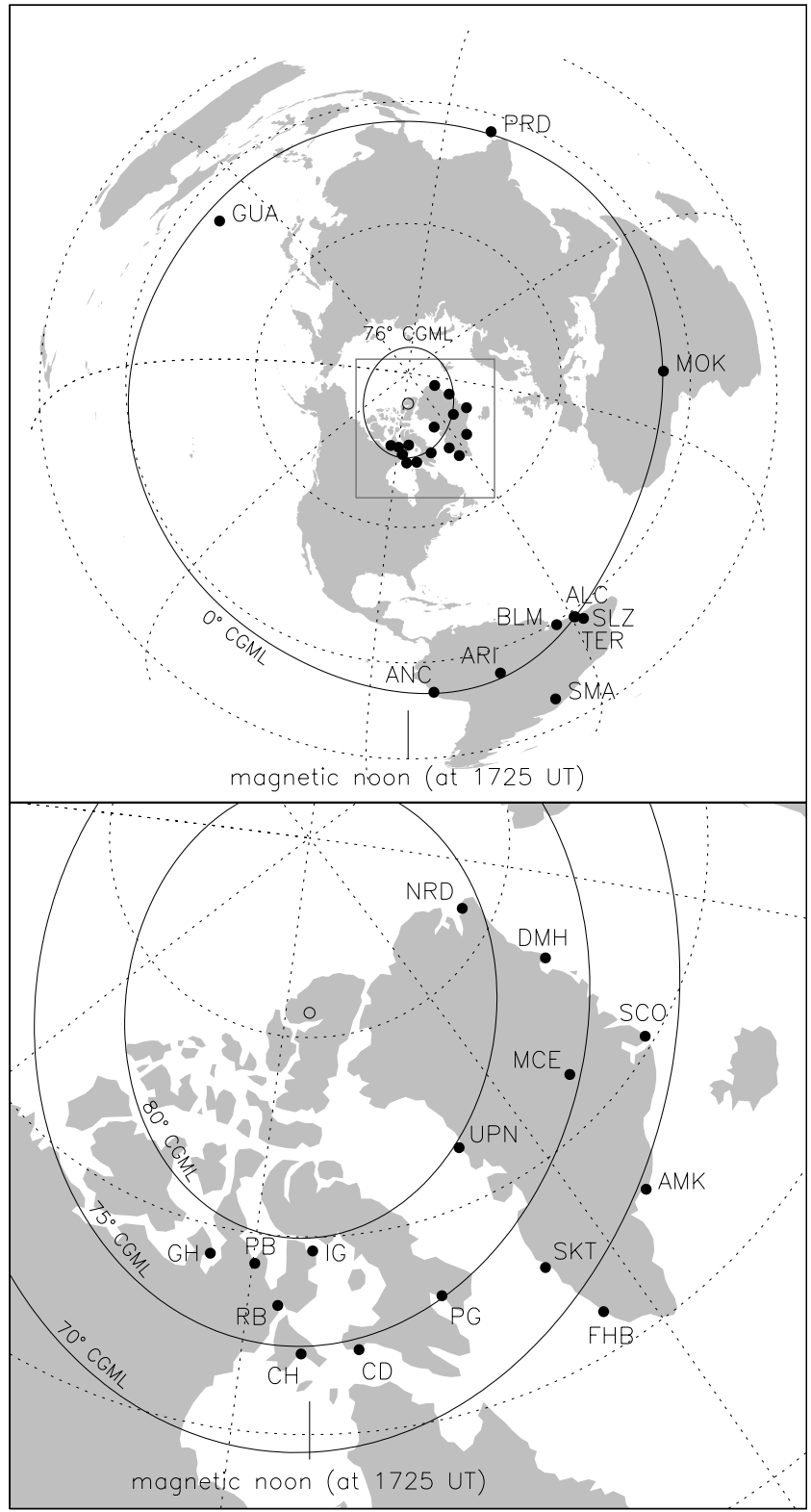

Figure 1. Locations of the Canadian, Greenland, and Equatorial ground magnetometers used in this study. The second panel presents an expanded view of the inset in the first panel.

but leave others disconnected, thereby delimiting the regions in which the spatially limited cavities can form.

[11] Although foreshock cavities are common [e.g., Sibeck et al., 2001], not all affect the magnetosphere. Because the IMF typically assumes a spiral orientation, such fluctuations must be far more common upstream from the prenoon than the postnoon bow shock. As noted by Greenstadt and Russell [1994], the wave-particle interactions required to generate foreshock cavities only begin to dominate the region upstream from the subsolar bow shock when the cone angle, $\Theta_{B n}$, between the IMF and Sun-Earth line falls below $\sim 51^{\circ}$. Since the dayside magnetosphere lies behind the subsolar bow shock, only those IMF fluctuations involving orientations below $51^{\circ}$ generate cavities that can subsequently influence the solar wind-magnetosphere interaction.
[12] Figure 3 illustrates the motion past the magnetosphere of three IMF bundles, each denoted as "B." Each bundle contains magnetic field lines that lie parallel to the ecliptic plane and point away from the Sun in the typical spiral direction. Broader regions in which the IMF points northward, as indicated by small circles, bound each bundle. Backstreaming ions appear within the bundles connected to the Earth's bow shock, and the pressure of these ions excavates cavities marked by depressed densities and magnetic field strengths (light gray shading in Figure 3). No backstreaming ions appear on the bounding northward magnetic field lines, which remain unconnected to the bow shock. However, the enhanced pressures within the expanding cavities compress the neighboring northward magnetic field regions (dark shading in Figure 3). Since spiral IMF field lines sweep across the magnetosphere from dusk to dawn, Figure 3 illustrates the progressive development of the cavities. Densities and magnetic field strengths within the cavities diminish (progressively lighter shading toward dawn), while densities and magnetic field strengths outside the cavities increase (progressively darker shading toward dawn). Furthermore, the cavities grow to extend further upstream from the bow shock with time.

[13] Figure 3 shows that IMP-8 was located upstream from the prenoon bow shock near GSE $(x, y, z)=(18,-25,0)$ $R_{E}$ during the interval from 1700 to 1800 UT on 9 November 1993. The IMP-8 plasma, magnetic field, and energetic ion observations presented in Figure 4 correspond closely to the predictions of the Thomas and Brecht simulation. Horizontal bars in the first panel indicate three periods when the $F$ detector on IMP-8 observed energetic ion counts exceeding

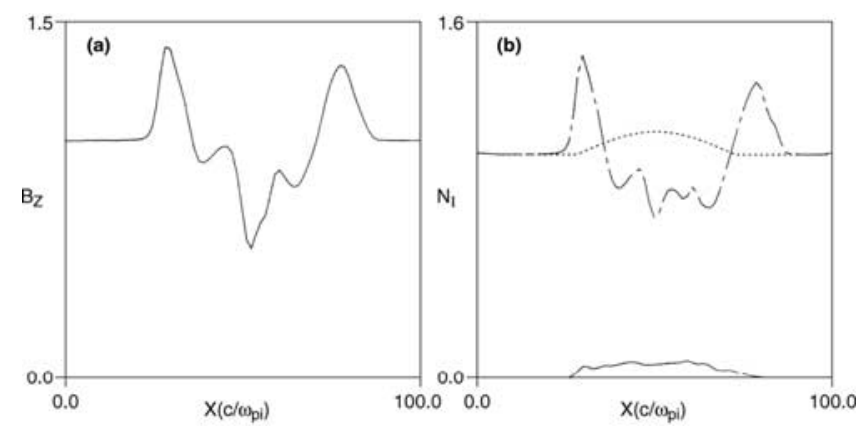

Figure 2. Enhanced pressure associated with the hot ion population formed by counterstreaming solar wind and suprathermal ion populations excavates a cavity of depressed densities and magnetic field strengths bounded by regions of enhanced densities and magnetic field strengths. The figure shows cross sections in the dawn/dusk direction for (a) the IMF strength $\left(B_{Z}\right)$ and (b) ion density $\left(n_{I}\right)$ across foreshock cavities [Thomas and Brecht, 1988]. It is to be noted that in this two-dimensional simulation, the $z$ direction points sunward along the Earth-Sun line and the magnetic field strengths and densities have been normalized to their unperturbed solar wind values. The dotted line in the second panel shows the initial profile for the combined solar wind and reflected populations, the dot-dash line shows the profile for the solar wind population at $\omega_{c i} t=43$, and the solid line at the base of the figure shows the profile for the suprathermal ions at $\omega_{c i} t=43$, where $\omega_{c i}$ is the ion cyclotron frequency. 


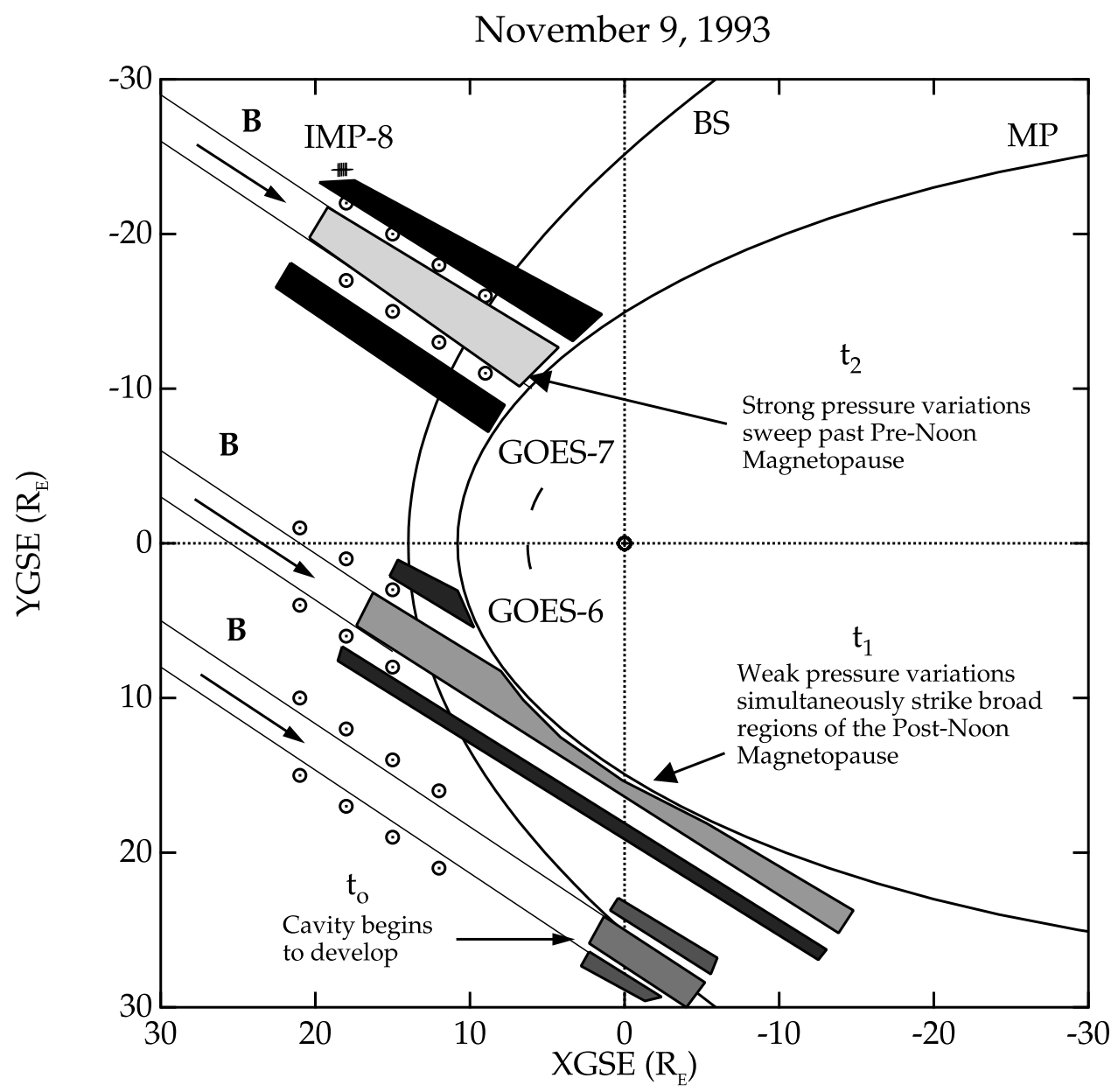

Figure 3. Idealized view of cavities forming on bundles of ecliptic magnetic field lines connected to the foreshock (light shading). The expanding cavities compress densities and magnetic field strengths on neighboring regions of northward magnetic field field lines unconnected to the bow shock (dark shading). The corresponding dynamic pressure variations convect to the bow shock, where they launch pressure variations that propagate across the magnetosheath to strike extended regions of the postnoon magnetopause nearly simultaneously before sweeping past the prenoon magnetopause. As time progresses, density and magnetic field strengths within the cavities diminish (lighter shading), while those outside increase (darker shading). The locations of IMP-8 in the solar wind, and GOES-6 and -7 in the magnetosphere, are shown.

200: $1719-1725,1727-1729$, and 1738-1743 UT. The second panel presents the IMF cone angle. Because the energetic ion bursts occur during intervals when $\Theta_{B n}<51^{\circ}$ any corresponding density and pressure variations will interact with the dayside magnetosphere. The third panel presents IMP-8 magnetic field observations in GSM coordinates, where longitude $\Phi=0^{\circ}$ points sunward, $90^{\circ}$ duskward, and latitude $\Theta=0^{\circ}$ lies in the equatorial plane. Enhanced fluctuations in the field orientation and a southward turning accompanied each energetic ion burst.

[14] The fourth and fifth panels of Figure 4 present IMP-8 plasma density and IMF strength observations, respectively. As the horizontal bars repeated in the fourth panel indicate, depressed densities and magnetic field strengths accompanied each energetic ion burst. Densities and magnetic field strengths reach greater than average values immediately adjacent to the cavities. Noting that in phase density and magnetic field strength variations are highly unusual in the pristine solar wind, Zesta et al. [1999] attributed the succession of perturbations on this day to processes occurring within the foreshock.

[15] We concur with the above report. The combined IMP8 energetic ion, plasma, and magnetic field observations decisively confirm this hypothesis and the predictions of the Thomas and Brecht [1988] model. The density and magnetic field strength decreases all corresponded to bursts of energetic ions. The prominent/prolonged density and magnetic field strength peaks at 1717-1719, 1724-1727, 1738, and 1744-1746 UT bounded ion bursts. Other more transient or weaker bursts occurred also near the edges of ion bursts.

\section{Interaction With the Equatorial Magnetosphere}

[16] Once they strike the bow shock, the upstream perturbations launch fast mode compression (and rarefaction) mode waves into the magnetosheath [Thomas et al., 1995]. During intervals of variable IMF orientation in which the 
November 9, 1993

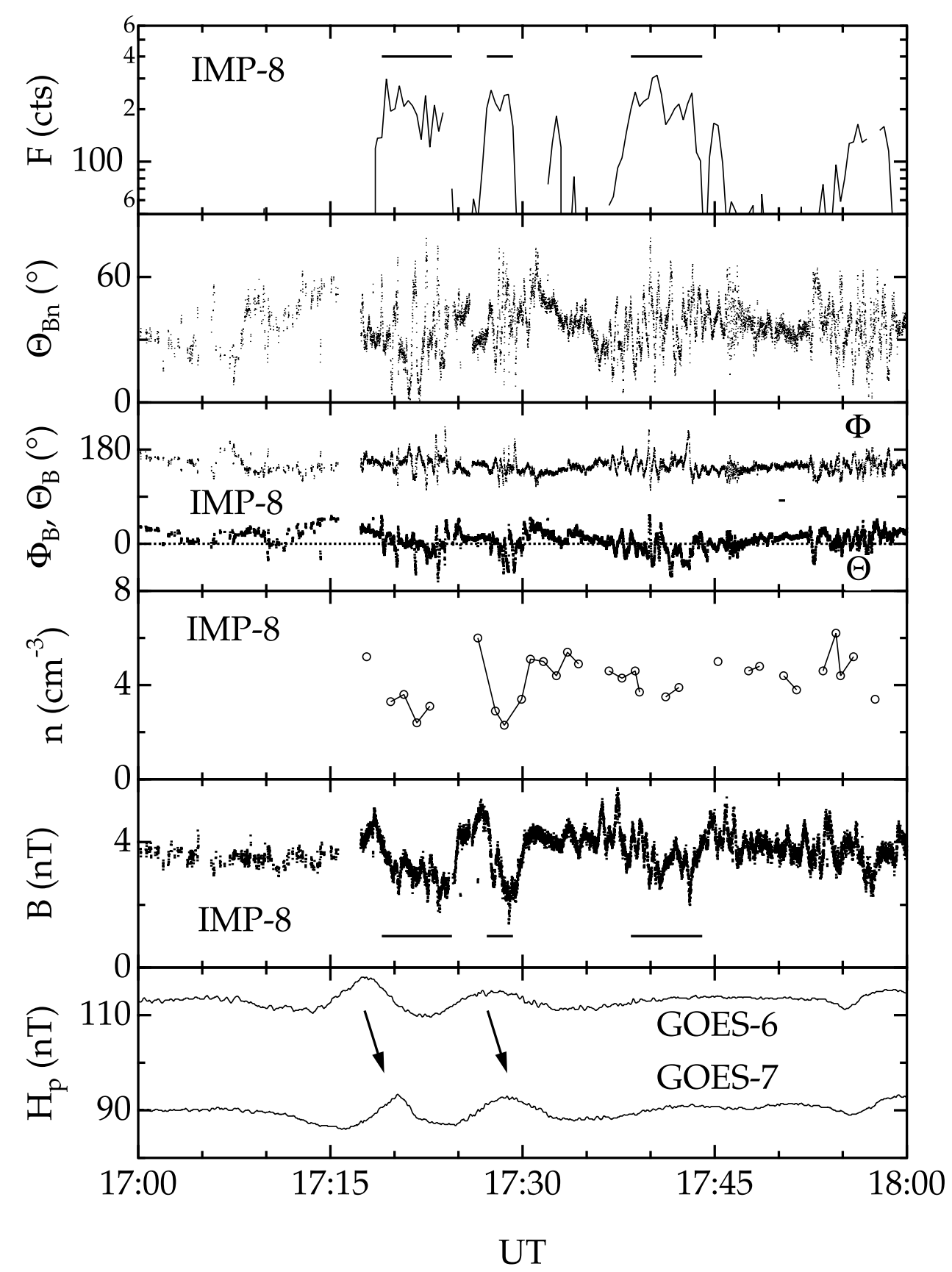

Figure 4. From top to bottom: IMP-8 EPE F detector energetic ion counts, IMP-8 IMF cone angle $\Theta_{B n}$ (defined as the angle between the IMF and the Sun-Earth line), IMP-8 IMF longitude and latitude in GSM coordinates, IMP-8 densities, IMP-8 IMF strengths, and the northward component of the geosynchronous magnetic field perpendicular to the equatorial plane observed by GOES-6 and -7 . Horizontal bars in the IMP-8 energetic ion flux and magnetic field strength panels indicate intervals when bursts of energetic ions exceeded 200 counts. The bars are repeated in the IMP- 8 magnetic field strength panel to illustrate the fact that they correspond to decreases in the magnetic field strength (and density). Arrows in the GOES geosynchronous magnetic field strength panel indicate the propagation of compressional signatures from GOES-6 to GOES-7.

angle between the IMF and the Sun-Earth lines falls below $\sim 51^{\circ}$, the dayside magnetosphere must be subjected to a constantly moving pressure differential [Greenstadt and Russell, 1994]. Because the sum of the fast mode and convection velocities within the magnetosheath is approximately the same as that in the solar wind, the transmitted pressure variations within the magnetosheath retain their solar wind alignment. Consequently, as illustrated in Figure 
3, pressure features aligned with the spiral IMF should strike broad regions of the postnoon magnetopause nearly simultaneously before sweeping dawnward across the dayside and prenoon magnetosphere.

[17] Upon reaching the magnetopause, magnetosheath pressure variations drive magnetopause motion. Invoking the fact that the quasi-parallel bow shock typically lies upstream from the prenoon magnetopause, both Sibeck [1990] and Fairfield et al. [1990] predicted that magnetopause motion attains far greater amplitude prior to local noon than afterward, a point confirmed by several previous [Howe and Siscoe, 1972; Wrenn et al., 1981; Rufenach et al., 1989] and one subsequent [Russell et al., 1997] study. During rarer periods of orthospiral IMF orientation, the foreshock moves to the region upstream from the postnoon bow shock and the amplitude of magnetopause motion increases on the postnoon magnetopause [Ivchenko et al., 2000].

[18] The pressure variations also launch fast and intermediate modes into the magnetosphere when they strike the magnetopause. Because the intermediate mode waves propagate solely along the magnetic field, only the fast mode waves reach spacecraft in the equatorial plane. Figure 3 shows equatorial projections of the duskward trajectories of postnoon GOES-6 and prenoon GOES-7 from 1700 to 1800 UT on 9 November 1993. As illustrated in the figure, we expect both the solar wind pressure variations and the fast mode waves they launch into the magnetosphere to sweep dawnward.

[19] The bottom panel of Figure 4 presents GOES-6 and -7 observations of the component of the geosynchronous magnetic field in the (northward) direction, perpendicular to the equatorial plane. As noted by Zesta et al. [1999], it is easy to identify magnetospheric compressions corresponding to the 1718 and $1725-1727$ UT inferred increases in the solar wind density/dynamic pressure. However, consistent with theoretical expectations for the more rapid decay of shorter-wavelength perturbations with distance from the magnetopause, it is impossible to identify responses at GOES- 6 or -7 to the other weaker or more transient inferred increases in the solar wind dynamic pressure. The dawnward motion of the compressional signatures from GOES-6 to GOES-7 confirms expectations that both events were produced by fast mode waves propagating dawnward through the magnetosphere.

\section{Interaction With the Equatorial Ionosphere}

[20] The fast mode waves seen at geosynchronous orbit propagate inward across magnetospheric magnetic field lines and eventually reach the equatorial ionosphere. We therefore expect signatures in equatorial ground magnetograms to resemble those at geosynchronous orbit. Furthermore, based on our discussion of Figure 3, we expect the IMF orientation to control the local time in the equatorial ionosphere where signatures originate, and their subsequent direction of motion. During typical periods of spiral IMF orientation, the fast mode waves launched by pressure variations should first strike the postnoon magnetopause, reach the postnoon equatorial ionosphere, and then spread to other local times. During more unusual periods with orthospiral IMF orientations, the signatures should first reach the prenoon magnetopause and ionosphere.
[21] From top to bottom, Figure 5 presents the $H$ components observed by GOES- 6 and several equatorial stations. A cubic fit over the period shown has been removed from each equatorial ground magnetogram to emphasize the shorter-period variations. As noted by Trivedi et al. [2002], MOK and the other equatorial stations recorded the two compressions observed by GOES-6 at 1718 and 1727 UT. The second, more clearly defined, compression peaks near 1728 UT at dusk (MOK), from 1729 to 1730 UT at postnoon local times (TER, ALC, SLZ, BLM, and SMA), and just after $1730 \mathrm{UT}$ at local noon (ANC, ARI), midnight (PRD), and early morning (GUA) local times. Consistent with expectations, the fast mode waves first reached the equatorial ionosphere near dusk and then propagated dawnward to other local times.

\section{Interaction With the High-Latitude Ionosphere}

\subsection{Theory}

[22] In contrast to the situation at the equator, both fast and intermediate mode waves reach the high-latitude ionosphere. Because the ionosphere is nearly incompressible, the convection excited by the transverse intermediate mode waves attains far greater amplitude than that excited by the compressional fast mode waves. Consequently, we must determine when and where intermediate mode waves and the field-aligned currents they carry are generated within the magnetosphere to determine the nature of the signatures expected in high-latitude ground magnetograms.

[23] Since the intermediate mode waves carry fieldaligned currents, the location and nature of the coupling can be determined by solving the momentum equation for the current parallel to the magnetic field [Vasyliunas, 1984]

$$
\mathbf{J}_{\|}=-\int_{S=0}^{S} \boldsymbol{\nabla}_{\perp} \cdot\left[\mathbf{B} \times\left(\rho d \mathbf{V} / d t+\nabla_{\perp} p\right) / B^{2}\right] d s
$$

in a specified geometry. As illustrated in Figure 6, we choose to consider the case of wavy motion $\left(A=A_{0} e^{i\left(\omega t-k_{y} y\right)+k_{x} x}\right)$ on a planar magnetopause in the presence of a LLBL. The $x$ component points in the direction normal to the nominal magnetopause, the $y$ component points duskward (or azimuthally) along the magnetopause in the equatorial plane, and the $z$ component points northward parallel to the zero-order magnetospheric magnetic field $\left(B_{0}\right)$. Sharp inner and outer edges delimit a uniform LLBL of stagnant magnetosheath-like plasma and depressed magnetospheric magnetic field strengths just inside the magnetopause.

[24] By contrast to Kivelson and Southwood [1991], we do not drop the pressure gradient term on the right-hand side of equation (1). By contrast to Glaßmeier and Heppner [1992], we do not drop the inertial term on the righthand side of equation (1). This enables us to obtain a simple, but exact, solution by linearizing equation (1), invoking the pressure balance condition for zero-order quantities $\left(p_{0}+B_{0}^{2} / 2 \mu_{0}=\right.$ constant $)$, using the polytropic gas law $\left(p_{0}=\alpha p_{0}^{\gamma}\right)$ and employing a moderate amount of algebraic manipulation:

$$
J_{1 \|}=\left(-2 B_{0}^{-2} \partial p_{0} / \partial x\right) \int_{z=0}^{z} \partial B_{1 z} / \partial y d z .
$$




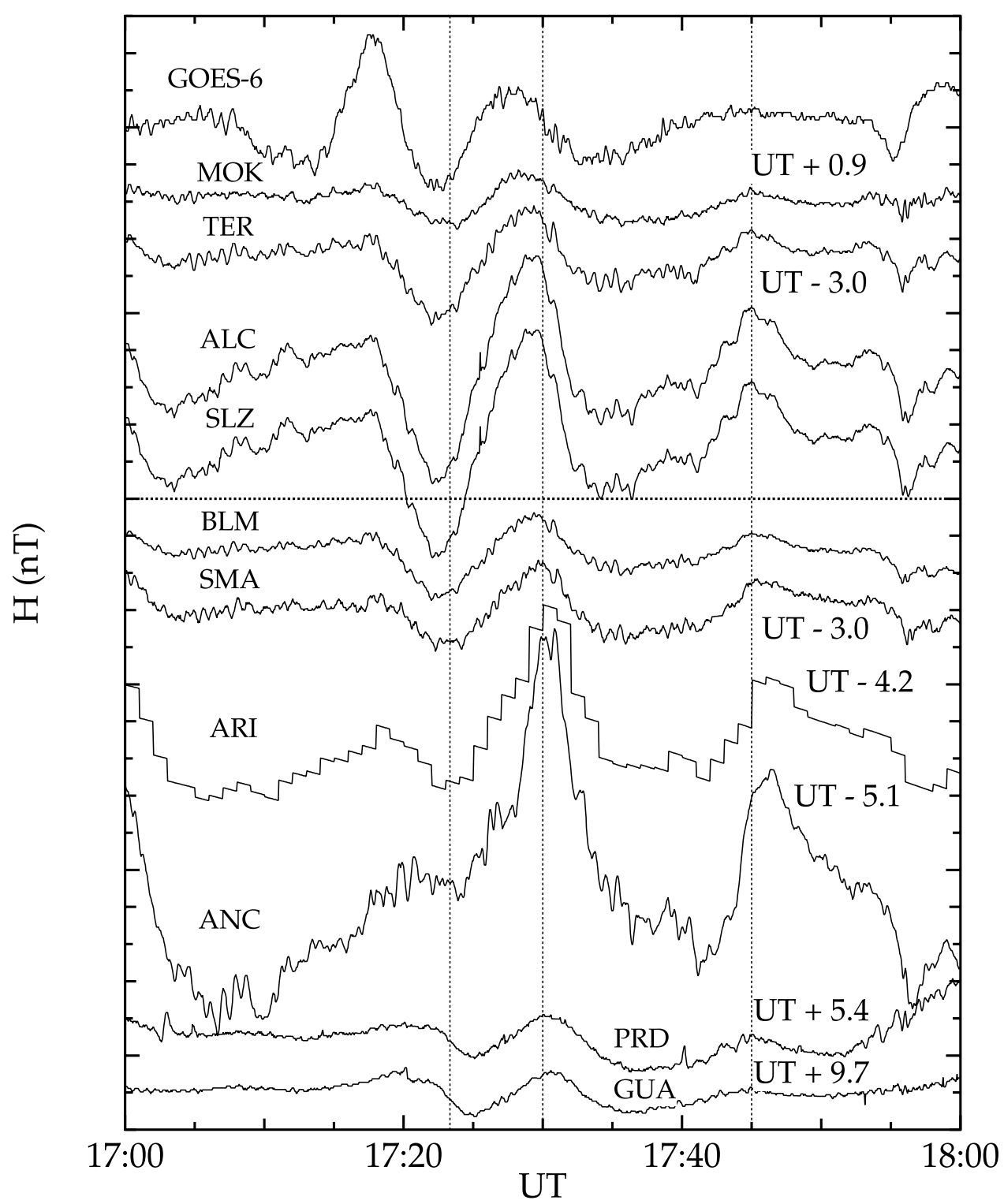

Figure 5. A comparison of the $H p$ component observed by GOES- 6 with the $X$ components of several equatorial ground magnetograms. The equatorial ground magnetograms are arranged from top to bottom versus local time, with MOK near dusk, TER, ALC, SLZ, BLM, SMA near 1400 LT, ARI near 1300 LT, and ANC near local noon, PRD near 2300 LT, and GUA near 0300 LT. Tick marks on the vertical axis are spaced 2 nT apart.

Since the field-aligned current actually results from pressure gradients applied to the magnetosphere, it is preferable (albeit more complicated) to express it in terms of the total pressure perturbation or $p_{1 T}\left(=p_{1}+B_{0} B_{1} / \mu_{0}\right)$ :

$$
\begin{aligned}
J_{1 \|}= & -2 B_{0}^{-2} \partial p_{0} / \partial x \int_{z=0}^{z}\left[B_{0} \rho_{0}^{-1}\left(C_{A}^{2}+C_{S}^{2}\right)^{-1}-\omega^{-2} k_{x} \partial B_{0} / \partial x\right] \\
& \cdot \partial p_{\mathrm{IT}} / \partial y d z
\end{aligned}
$$

where the first term in the brackets accounts for changes in the magnetic field strength by compressions and the second for changes caused by the passage of zero-order gradients.

[25] As was the case in the solutions obtained by Kivelson and Southwood [1991] and Glaßmeier and Heppner [1992], equation (3) indicates that discrete field-aligned currents arise only when azimuthal pressure perturbations are applied to radial pressure (or equivalent density or magnetic field strength) gradients. Consistent with the predictions of Glaßmeier and Heppner [1992], but in contrast to those of Southwood and Kivelson [1990] and Lysak et al. [1994], equation (3) predicts that each transient indentation in the magnetopause produces a pair of azimuthally spaced and oppositely directed field-aligned currents on field lines mapping to sharp gradients at both the magnetopause and the inner edge of the LLBL. Since both terms in the brackets on the right-hand side of equation ( 3 ) are positive $\left(\partial B_{0} / \partial x<0\right)$, the total field-aligned current $J_{1 \|}$ is negative when there is a duskward gradient in the total pressure, and positive when there is dawnward gradient. Consequently, the sense of the 


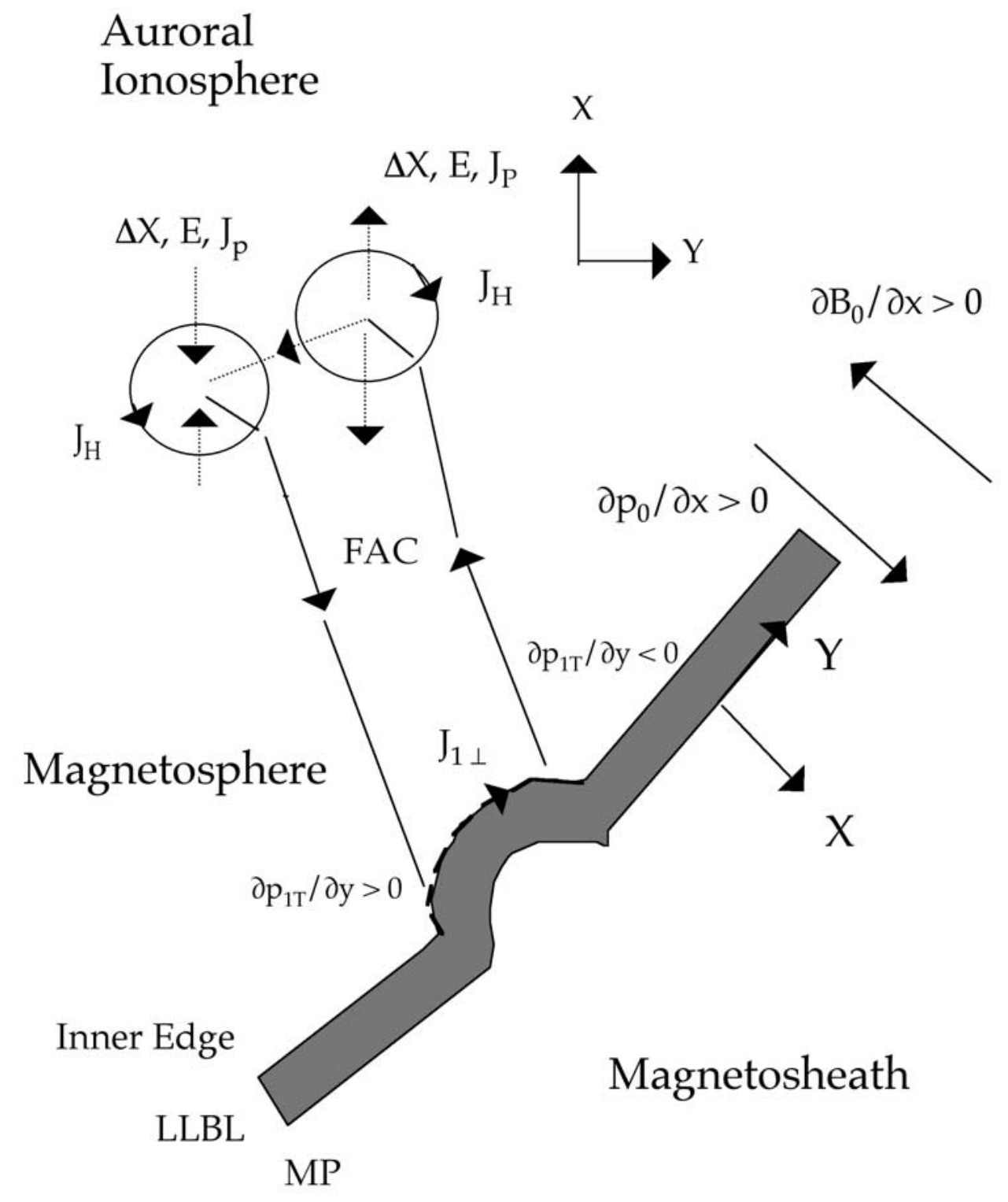

Figure 6. The generation of field-aligned currents at the inner edge of the LLBL. Each transient compression of the magnetopause generates a pair of oppositely directed field-aligned currents on the inner edge of the LLBL. Hall current vortices associated with the field-aligned currents generate the signatures observed by magnetometers in the high-latitude ionosphere.

field-aligned currents generated by pressure enhancements applied to the magnetosphere is the same as that illustrated by Glaßmeier and Heppner [1992], namely upward from the ionosphere on the dawnside of the enhancement, and downward into the ionosphere on the duskside of the enhancement. As explained by Glaßmeier and Heppner [1992], the field-aligned currents are precisely those required to close the increases (and decreases) in the perpendicular currents on the boundaries of the LLBL that occur during transient compressions (and expansions) of the magnetopause (see Figure 6).

[26] Because magnetopause magnetic field lines map to the cusp, a relatively narrow region in the ionosphere, it is nearly impossible to identify or track the motion of TCVs produced by current perturbations on the magnetopause. By contrast, the locus of points corresponding to the inner edge of the magnetospheric LLBL maps to a broad array of local times [Newell and Meng, 1992], making it a relatively simple matter to track the azimuthal motion of TCVs on the equatorward edge of the ionospheric LLBL.

[27] Individual TCVs can be identified on the basis of monopolar magnetic field perturbations in ground magnetometer arrays. As illustrated in Figure 6, Pedersen currents and electric fields (dashed arrows) point inward toward upward field-aligned currents, but outward from downward field-aligned currents. Hall currents (solid circles) flowing perpendicular to these induced electric fields produce magnetic field perturbations (the same dashed arrows) that point inward toward upward field-aligned currents, but outward away from downward field-aligned currents. As illustrated by Zesta et al. [2002], stations under the northern fringes of vortices produced by upward field-aligned currents observe 
southward magnetic field perturbations, while those under the southern fringes observe northward perturbations. Perturbations in the opposite sense occur during the passage of vortices associated with downward field-aligned currents.

\subsection{Observations}

[28] We can tailor the predictions of section 6.1 to the observations presently under study. Pressure variations aligned with the spiral IMF strike broad regions of the postnoon magnetopause nearly simultaneously, then sweep dawnward across the prenoon magnetopause. Consequently, they should apply little or no azimuthal pressure gradients to broad regions of the postnoon magnetopause, generate no field-aligned currents in the postnoon magnetosphere, and produce no TCVs in the postnoon high-latitude ionosphere. By contrast, each of the two main compressions indicated by arrows at 1718 and 1728 UT in the lower panel of Figure 4 should apply azimuthal pressure gradients to the prenoon magnetosphere, briefly compress and enhance the azimuthal currents on the inner edge of the prenoon LLBL, generate a pair of oppositely directed field-aligned currents, and produce a pair of oppositely rotating TCVs on prenoon magnetic field lines that map to the inner edge of the LLBL. The leading TCV in each dawnward moving pair should be associated with an upward field-aligned current and the trailing TCV with a downward field-aligned current.

[29] At a minimum, transient events in the ionosphere must move azimuthally and exhibit reversal in magnetic field perturbations with latitude to be identified as TCVs.

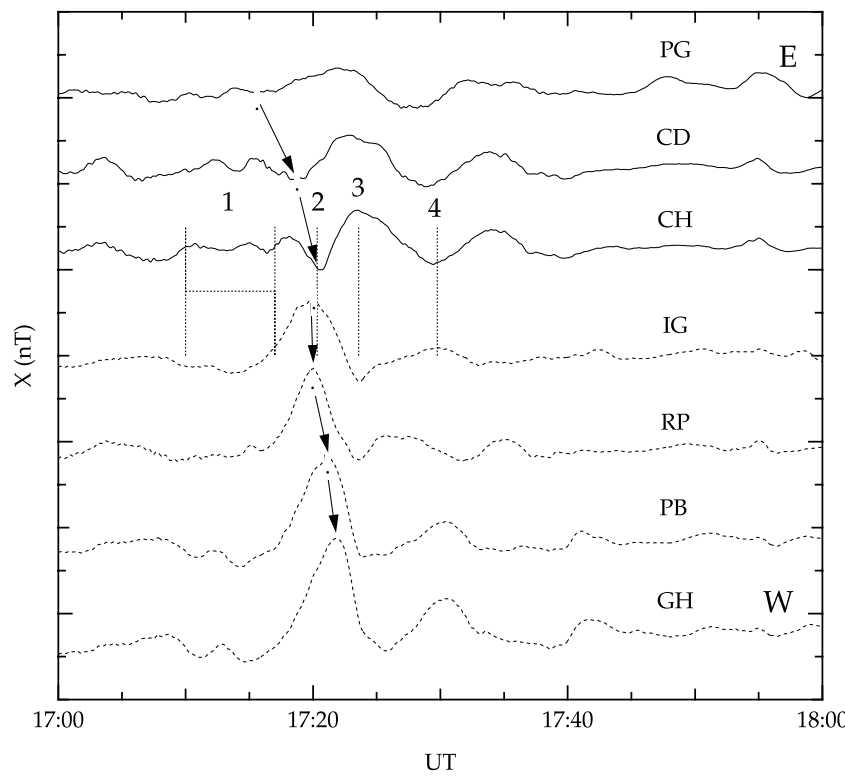

Figure 7. The $X$ (geographic northward) components of MACCS array ground magnetograms from 1700 to 1800 UT on 9 November 1993. The traces are displayed from east (E) to west (W) in two groups: solid lines indicate those at lower latitudes, whereas dotted lines indicate those at higher latitudes. Four vertical dashed lines and numbers indicate times when anticorrelated $X$ components at $\mathrm{CH}$ and $\mathrm{IG}$ provide evidence for reversals in the azimuthal convection with latitude, Hall current vortices, and the passage of fieldaligned currents. Tick marks on the vertical axis are spaced $100 \mathrm{nT}$ apart.

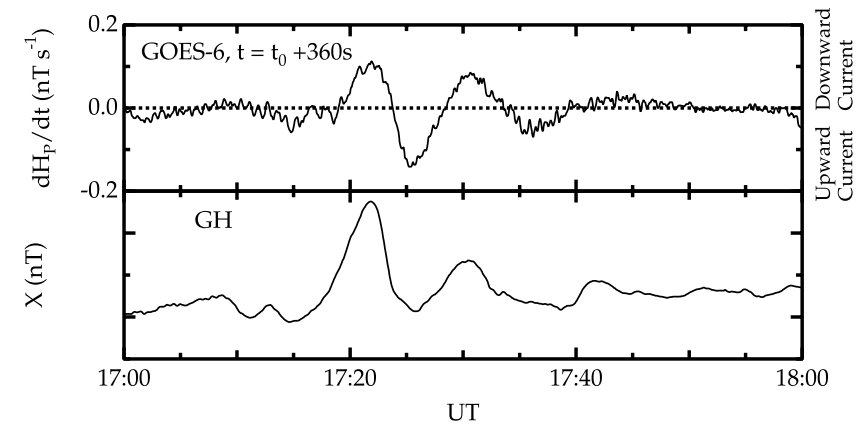

Figure 8. A qualitative comparison of the field-aligned current strength and direction predicted from (a) the inferred azimuthal gradient in the magnetospheric pressure (proportional to the time derivative of the GOES-6 geosynchronous magnetic field strength) and (b) the north/south perturbations induced in the GH ground magnetogram. The GOES-6 observations have been lagged by $360 \mathrm{~s}$. Tick marks on the vertical axis of the GH panel are spaced 100 nT apart.

Figure 7 presents observations of the $X$ (geographic north) components seen by the MACCS magnetometers from 1700 to 1800 UT on 9 November 1993. The observations have been divided into two groups: those from geomagnetic latitudes below $75^{\circ}(\mathrm{PG}, \mathrm{CD}$, and $\mathrm{CH})$ and those from latitudes above $76.5^{\circ}$ (IG, RB, PB, and $\left.\mathrm{GH}\right)$. As the arrows indicate, individual perturbations from 1715 to 1745 UT moved dawnward at both low and high latitudes, from PG to $\mathrm{CH}$ and from IG to GH. Zesta et al. [1999] estimated the overall azimuthal speed of the various features during this interval as $\sim 3.6 \mathrm{~km} \mathrm{~s}^{-1}$.

[30] Zesta et al. [1999] applied a 30 min high-pass filter to the observations to remove the background convective flow and isolate the signatures of the four TCVs identified by vertical dashed lines in Figure 7 . Because they are aligned nearly along a magnetic meridian, $\mathrm{IG}$ and $\mathrm{CH}$ are an appropriate station pair to identify transient signatures in the $X$ component whose sense reverses over the geomagnetic latitude range from $74.6^{\circ}$ to $79.4^{\circ} \mathrm{N}$. From 1710 to 1717 UT and at 1723 UT, IG observed weak southward perturbations and $\mathrm{CH}$ observed weak northward perturbations. Since the perturbations pointed inward toward a common center lying between the stations, they were produced by upward field-aligned currents. At 1720 and 1730 UT, IG observed northward perturbations and $\mathrm{CH}$ observed southward perturbations. Since the perturbations pointed outward away from a common center, these events were produced by downward field-aligned currents. Consequently, the ground magnetometer observations can be interpreted as an evidence for the passage of two pairs of oppositely directed field-aligned currents, with the upward current leading in each case, precisely the scenario predicted above.

[31] Like Zesta et al. [1999], we associated the TCVs with transient compressions observed by the GOES spacecraft. To strengthen this argument, Figure 8 compares estimates of the field-aligned currents based on magnetospheric and ionospheric measurements. As indicated by equation (3), field-aligned current strengths are proportional to azimuthal pressure gradients, which can in turn be computed from time derivatives of the GOES- 6 magnetic 


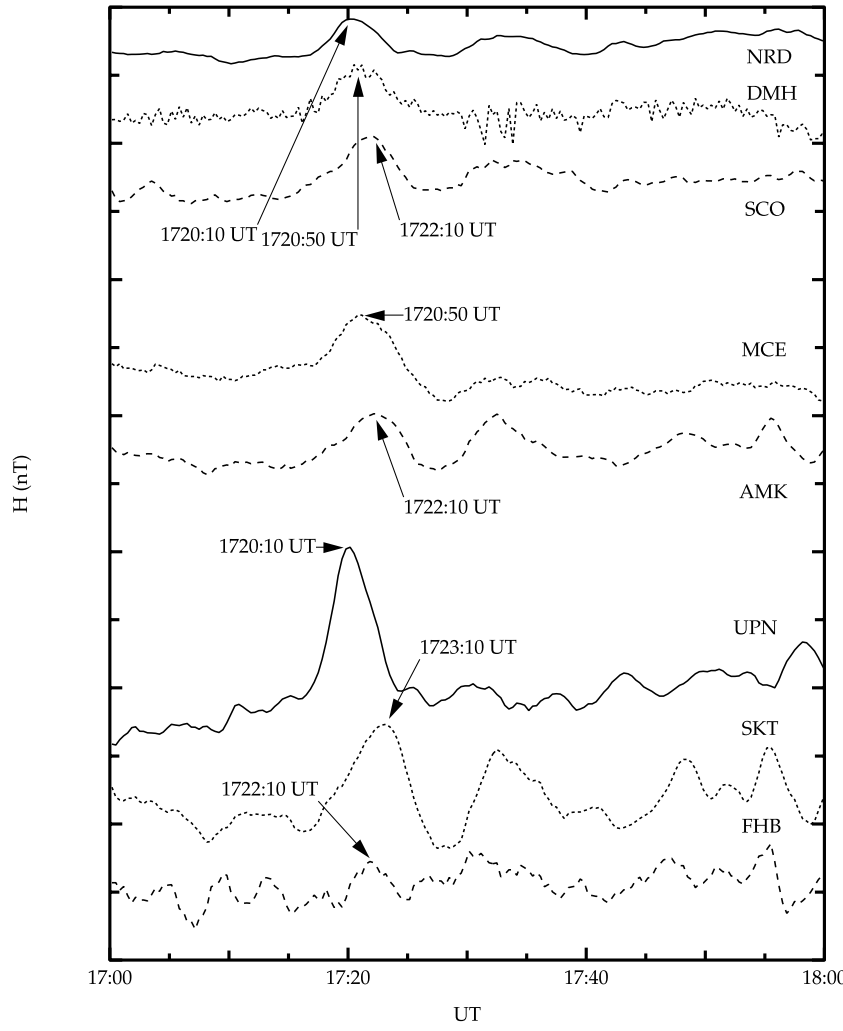

Figure 9. The $H$ (northward) components of Greenland ground magnetograms from 1700 to 1800 UT on 9 November 1993. The observations are organized by latitude within each of three local time groups. Arrows indicate times when the $H$ component peaks. Tick marks on the vertical axis are spaced $25 \mathrm{nT}$ apart.

field strength under the twin assumptions that the contribution of the plasma to the total pressure within the magnetosphere is negligible and that the transient compressions move dawnward at a constant velocity $V_{0}$, i.e., that $-J_{1 \|}$ $\sim \partial p_{T} / \partial y \sim \partial B_{\mathrm{P}} / \partial y \sim V_{0}^{-1} \partial B_{\mathrm{P}} / \partial t$ [Sibeck and Korotova, 2000]. Figure $8 \mathrm{a}$ presents the field-aligned current variations determined from GOES- 6 observations. For comparison, we present estimates of the field-aligned current strength and direction determined from a ground magnetogram. Figure $8 \mathrm{~b}$ presents $X$ component observations from $\mathrm{GH}$, located northward from the vortex centers. At this location, southward perturbations indicate upward currents and northward perturbations indicate downward currents in the ionosphere. Observationally, we find that the GOES-6 and ground observations correspond best when the GOES-6 observations are lagged by $6 \mathrm{~min}$. The similarity of the traces in Figures $8 \mathrm{a}$ and $8 \mathrm{~b}$ confirms the model for fieldaligned currents derived and presented in this paper.

[32] The TCVs should lie centered upon the equatorial boundary of field lines mapping to the LLBL. During the time interval under study here, the DMSP-F10 spacecraft flew through the auroral oval in the southern hemisphere at prenoon local times, i.e., passed over the conjugate fooprints of field lines mapping to the MACCS. According to the region identification scheme derived by Newell and Meng [1992] for DMSP particle flux observations, the spacecraft observed the LLBL from geomagnetic latitude $-74.2^{\circ}$ and $1053 \mathrm{LT}$ at $1737: 51 \mathrm{UT}$ to geomagnetic latitude $-81.1^{\circ}$ and $1154 \mathrm{LT}$ at $1740 \mathrm{UT}$ (not shown). By using all the MACCS array stations, we can pin down the latitude at which the sense of the perturbation in the $X$ component reverses to somewhere between $75.2^{\circ}(\mathrm{PG})$ and $76.9^{\circ}(\mathrm{RB})$. Note that the transient nature of the event signatures, and the fact that they can be observed in the filtered observations, ensure that they cannot be interpreted in terms of the convection reversal boundary commonly encountered near the equatorward edge of the LLBL. Consequently, the DMSP observations confirm that the centers of the vortices occurred on or near-magnetic field lines that map deep within the magnetospheric LLBL.

[33] Despite the fact that their latitudinal coverage exceeds $10^{\circ}$, the postnoon Greenland ground magnetograms presented in Figure 9 provide no evidence for any convection reversal with latitude. The observations have been organized into three groups: the three upper traces present observations from the east coast near 1800 MLT, the two middle traces present observations from central Greenland at 1700 MLT, and the three lower traces present observations from the west coast at 1500 MLT. Within each group, the traces are ordered from north to south. Similar perturbations were seen at all latitudes. In contrast to the much stronger perturbations seen in the MACCS array, amplitudes at Greenland stations only reached $\sim 50 \mathrm{nT}$.

[34] Nor do the Greenland observations provide any evidence for azimuthal motion. Near $79.5^{\circ}$, postnoon UPN and nightside NRD (solid lines in Figure 9) observed peaks in the $H$ component nearly simultaneously at 1720:10 UT. Near $74^{\circ}$, nightside DMH and late afternoon MCE near $74^{\circ}$ observed this peak at 1720:50 UT. At latitudes from $68^{\circ}$ to $72^{\circ}$, nightside to midafternoon stations SCO, AMK, and FHB (dashed lines in Figure 9) observed the peak at 1722:10 UT. If anything, rather than propagating azimuthally, the signatures observed in the Greenland ground magnetograms propagated equatorward. We conclude that no TCVs were seen in the high-latitude postnoon ionosphere, consistent with model predictions.

\section{Discussion and Conclusions}

[35] We presented a detailed comparison of solar wind, magnetospheric, and ionospheric observations with models during an interval in which four TCVs were detected propagating dawnward through the high-latitude prenoon ionosphere. The ground events had previously been associated with two pressure pulses seen in the solar wind. We compared energetic ion, plasma, and magnetic field observations of these pressure pulses with the predictions of a hybrid code mode for the interaction of reflected ions with the solar wind in the foreshock. As predicted by the model, pressures associated with the bursts of energetic ions excavated cavities in the solar wind density and magnetic field strength. The excavated material piled up on the edges of the cavities.

[36] The significance of these observations lies in the fact that theory predicts [Terasawa, 1979], and observations confirm [Lin et al., 1974], that the flux of energetic ions decays rapidly with distance from the bow shock. Consequently, the plasma density and dynamic pressure perturbations generated by the ions can only be observed 
immediately upstream from the quasi-parallel bow shock. Spacecraft in other locations may observe fluctuations in the IMF orientation [e.g., Konik et al., 1994], but not the pressure variations generated within the foreshock.

[37] The foreshock pressure variations launched dawnward-moving waves on the magnetopause and dawnwardmoving compressions at geosynchronous orbit. Consistent with model predictions, we used high-time resolution observations to demonstrate that the same fast mode waves also propagated dawnward through the equatorial ionosphere from a point of origin near dusk. Given the typical spiral IMF orientation, we would generally expect compressional SI signatures to originate at postnoon local times. In fact, Nishida and Jacobs [1962] presented (without explanation) the results of a statistical survey of low-latitude ground magnetograms confirming that this is precisely the case.

[38] Without making restrictive assumptions, we derived a relationship between field-aligned currents and pressure perturbations applied to the magnetopause. Like previous researchers, we concluded that field-aligned currents and ionospheric TCVs would be generated when and where transient azimuthal pressure gradients are applied to the more permanent radial gradients. In contrast to some previous models, we concluded that each pressure pulse applied to the magnetosphere would produce a pair of oppositely directed field-aligned currents, and oppositely circulating TCVs, on the inner edge of the LLBL. We showed that the inferred sense of the field-aligned currents producing the sequence of four TCVs on 9 November 1993 reported by Zesta et al. [1999] was consistent with that expected by our model for the two pressure pulses driving these events.

[39] The results of this study offer clues to interpreting those from previously reported statistical surveys. Because there are no solar wind dynamic pressure variations corresponding to most quasi-periodic TCVs and the centers of these events occur on magnetic field lines that map to the convection reversal at the inner edge of the LLBL, McHenry et al. [1990], Clauer and Ridley [1995], and Clauer et al. [1997] concluded that they most likely occur in response to the Kelvin-Helmholtz instability occurring on this boundary. However, the results of the present survey suggest that pressure pulses generated within the foreshock can be an important cause of TCVs and indicate that the signatures of events generated by pressure pulses should also peak at the inner edge of the LLBL. A renewed effort to determine whether some quasi-periodic TCVs can be associated with variations in the IMF orientation during periods when the IMF cone angle is small is clearly warranted.

[40] Finally, the models predict no TCV generation in the postnoon ionosphere during typical periods of spiral IMF orientation. In fact, statistical surveys show that events are far more common in the prenoon ionosphere [Glaßmeier et al., 1989; Lanzerotti et al., 1991]. An effort to determine whether TCVs occur preferentially in the postnoon ionosphere during rarer periods of orthospiral IMF orientation is now necessary.

[41] Acknowledgments. D.G. Sibeck and J. Watermann thank the International Space Institute in Bern, Switzerland for their hospitality during the time period when this work began. D.G. Sibeck acknowledges with gratitude helpful conversations with $\mathrm{M}$. Hesse and the extensive comments of both referees. This research was funded by NSF grant ATM-0096534 with a supplement from NSF/INT. IMP-8 observations were supplied by the principal investigators to the NSSDC, thence to us. The MACCS project is managed jointly by Augsburg College and Boston University with support from NSF grants ATM-9111727, ATM-ATM9401524, ATM-9401733, ATM-9610072, ATM-9704766, and ATM0000339. The Danish Meteorological Institute supplied the Greenland ground magnetometer observations, and the equatorial magnetometer observations were provided by Kyushu University. MAGIC-1 East observations were obtained as part of the MAGIC project by the Space Physics Research Laboratory at the University of Michigan with support from NSF grants ATM-9204520, ATM-9501380, OPP-9318766, OPP-9614175, OPP9614189, OPP-9801941, OPP-9876473, and OPP-0220735.

[42] Lou-Chang Lee and Chin S. Lin thank C. Robert Clauer and Mark J. Engebretson for their assistance in evaluating this paper.

\section{References}

Clauer, C. R., and A. J. Ridley, Ionospheric observations of magnetospheric low-latitude boundary-layer waves on August 4, 1991, J. Geophys. Res., 100, 21, 873-21,884, 1995

Clauer, C. R., A. J. Ridley, R. J. Sitar, H. J. Singer, A. S. Rodger, E. FriisChristensen, and V. O. Papitashvili, Field line resonant pulsations associated with a strong dayside ionospheric shear convection flow reversal, J. Geophys. Res., 102, 4585-4596, 1997.

Fairfield, D. H., W. Baumjohann, G. Paschmann, H. Lühr, and D. G. Sibeck, Upstream pressure variations associated with the bow shock and their effects on the magnetosphere, J. Geophys. Res., 95, 37733786, 1990.

Friis-Christensen, E., M. A. McHenry, C. R. Clauer, and S. Vennerstrom, Ionospheric traveling convection vortices observed near the polar cleft: A triggered response to sudden changes in the solar wind, Geophys. Res. Lett., 15, 253-256, 1988.

Glaßmeier, K.-H., and C. Heppner, Traveling magnetospheric convection twin vortices-Another case study, global characteristics, and a model, J. Geophys. Res., 97, 3977-3992, 1992.

Glaßmeier, K.-H., M. Honish, and J. Untiedt, Ground-based and satellite observations of traveling convection twin vortices, J. Geophys. Res., 94, $2520-2528,1989$.

Greenstadt, E. W., and C. T. Russell, Stimulation of exogenic, daytime geomagnetic pulsations: A global perspective, in Solar Wind Sources of Magnetospheric Ultra-Low-Frequency Waves, Geophys. Monogr. Ser., vol. 81, edited by M. J. Engebretson, K. Takahashi, and M. Scholer, pp. 13-23, AGU, Washington, D. C., 1994.

Greenstadt, E. W., I. M. Green, D. S. Colburn, J. H. Binsack, and E. F. Lyon, Dual satellite observations of Earth's bow shock, part II, Field aligned upstream waves, Cosmic Electrodyn., 1, 279-287, 1970.

Howe, H. C., and G. L. Siscoe, Magnetopause motions at lunar distance determined from the explorer 35 plasma experiment, J. Geophys. Res., 77, 6071-6086, 1972.

Hughes, W. J., and M. J. Engebretson, MACCS: Magnetometer array for cusp and cleft studies, in The Satellite-Ground Based Coordination Sourcebook, ESA-SP-1198, edited by M. Lockwood, M. N. Wild, and H. J. Opgenoorth, pp. 119-123, Eur. Space Agency Publ., ESTEC, Noordwijk, Netherlands, 1997.

Ivchenko, N. V., D. G. Sibeck, K. Takahashi, and S. Kokubun, A statistical study of the magnetosphere boundary crossings by the geotail satellite, Geophys. Res. Lett., 27, 2881-2884, 2000.

King, J. H., Availability of IMP-7 and IMP-8 data for the IMS period, in The IMS Source Book, edited by C. T. Russell and D. J. Southwood, pp. 10-20, AGU, Washington, D. C., 1982.

Kivelson, M. G., and D. J. Southwood, Ionospheric traveling vortex generation by solarwind buffeting of the magnetosphere, J. Geophys. Res., 96, 1661-1667, 1991 .

Konik, R. M., L. J. Lanzerotti, A. Wolfe, C. G. Maclennan, and D. Venkatesan, Cusp latitude impulse events, 2, Interplanetary magnetic field and solar wind conditions, J. Geophys. Res., 99, 14,831-14,853, 1994.

Korotova, G. I., D. G. Sibeck, T. Moretto, and G. D. Reeves, Tracking transient events through geosynchronous orbit, J. Geophys. Res., 104, $10,265-10,273,1999$

Korotova, G. I., D. G. Sibeck, H. J. Singer, and T. J. Rosenberg, Tracking transient events through geosynchronous orbit and in the high-latitude ionosphere, J. Geophys. Res., 107, 1345, doi:10.1029/2002JA009477, 2002.

Lanzerotti, L. J., L. C. Lee, C. G. Maclennan, A. Wolfe, and L. V. Medford, Possible evidence of flux transfer events in the polar ionosphere, Geophys. Res. Lett., 13, 1089-1092, 1986.

Lanzerotti, L. J., R. M. Konik, A. Wolfe, D. Venkatesan, and C. G. Maclennan, Cusp latitude magnetic impulsive events, 1, Occurrence statistics, J. Geophys. Res., 96, 14,009-14,022, 1991. 
Lin, R. P., C.-I. Meng, and K. A. Anderson, 30- to 100-keV protons upstream from the Earth's bow shock, J. Geophys. Res., 79, 489-498, 1974

Lysak, R. L., Y. Song, and D. H. Lee, Generation of ULF waves by fluctuations in the magnetopause position, in Solar Wind Sources of Magnetospheric ULF Waves, Geophys. Monogr. Ser, vol. 81, edited by M. J. Engebretson, K. Takahashi, and M. Scholer, pp. 273-281, AGU, Washington, D. C., 1994.

McHenry, M. A., C. R. Clauer, E. Friis-Christensen, and J. D. Kelly, Observations of ionospheric convection vortices: Signatures of momentum transfer, Adv. Space Res., 8, 315-320, 1988

McHenry, M. A., C. R. Clauer, and E. Friis-Christensen, Relationship of solar wind parameters to continuous, dayside, high latitude traveling ionospheric convection vortices, J. Geophys. Res., 95, 15,007-15,022, 1990.

Newell, P. T., and C.-I. Meng, Mapping the dayside ionosphere to the magnetosphere according to particle precipitation characteristics, Geophys. Res. Lett., 19, 609-612, 1992.

Nishida, A., and J. A. Jacobs, World-wide changes in the geomagnetic field, J. Geophys. Res., 67, 525-539, 1962.

Paschmann, G., N. Sckopke, I. Papamastorakis, J. R. Asbridge, S. J. Bame, and J. T. Gosling, Characteristics of reflected and diffuse ions upstream from the Earth's bow shock, J. Geophys. Res., 86, $4355-$ 4364, 1981

Rufenach, C. L., R. F. Martin Jr., and H. H. Sauer, A study of geosynchronous magnetopause crossings, J. Geophys. Res., 94, 15,125-15,134 1989.

Rufenach, C. L., J. Schaper, and R. L. McPherron, The quiet geomagnetic field at geosynchronous orbit and its dependence on solar wind dynamic pressure, J. Geophys. Res., 97, 25-42, 1992.

Russell, C. T., S. M. Petrinec, T. L. Zhang, P. Song, and H. Kawano, The effect of foreshock on the motion of the dayside magnetopause, Geophys. Res. Lett., 24, 1439-1442, 1997.

Sibeck, D. G., A model for the transient magnetospheric response to sudden solar wind dynamic pressure variations, J. Geophys. Res., 95, 37553771, 1990.

Sibeck, D. G., Transient magnetic field signatures at high latitudes, J. Geophys. Res., 98, 243-256, 1993.

Sibeck, D. G., and G. I. Korotova, Occurrence patterns for transient magnetic field signatures at high latitudes, J. Geophys. Res., 101, 13,413$13,428,1996$

Sibeck, D. G., and G. I. Korotova, Testing models for traveling convection vortices: Two case studies, Geophys. Res. Lett., 27, 325-328, 2000

Sibeck, D. G., W. Baumjohann, and R. E. Lopez, Solar wind dynamic pressure variations and transient magnetospheric signatures, Geophys. Res. Lett., 16, 13-16, 1989.

Sibeck, D. G., R. B. Decker, D. G. Mitchell, A. J. Lazarus, R. P. Lepping, and A. and Szabo, ind preconditioning in the flank foreshock: IMP 8 observations, J. Geophys. Res., 106, 21,675-21,688, 2001.
Southwood, D. J., and M. G. Kivelson, netohydrodynamic response of the magnetospheric cavity to changes in solar wind pressure, J. Geophys. Res., 95, 2301-2309, 1990.

Tachihara, H., M. Shinohara, M. Shimoizumi, O. Saka, and T. Kitamura, Magnetometer system for studies of the equatorial electrojet and micropulsations in equatorial regions, J. Geomagn. Geoelectr., 48, 1311-1320, 1996.

Terasawa, T., Origin of $30 \sim 100 \mathrm{keV}$ protons observed in the upstream region of the Earth's bow shock, Planet. Space Sci., 27, 365-384, 1979.

Thomas, V. A., and S. H. Brecht, Evolution of diamagnetic cavities in the solar wind, J. Geophys. Res., 93, 11,341-11,353, 1988.

Thomas, V. A., D. Winske, and M. F. Thomsen, Simulation of upstream pressure pulse propagation through the bow shock, J. Geophys. Res., 100, 23,481-23,488, 1995

Trivedi, N. B., D. G. Sibeck, E. Zesta, J. C. Santos, K. Yumoto, T. Kitamura, M. Shinohara, and S. L. G. Dutra, Signatures of traveling convection vortices in ground magnetograms under the equatorial electrojet, J. Geophys. Res., 107, 1087, doi:10.1029/2001JA000153, 2002.

Vasyliunas, V. M., Fundamentals of current description, in Magnetospheric Currents, Geosphys. Monogr. Ser, vol. 28, edited by T. A. Potemra, pp. 63-66, AGU, Washington, D. C., 1984.

Wilhjelm, J., and E. Friis-Christensen, Magnetometer chain in Greenland, Geophys. Pap. R-48, Dan. Meteorol. Inst., Copenhagen, 1976.

Wrenn, G. L., J. F. E. Johnson, A. J. Norris, and M. F. Smith, GEOS-2 magnetopause encounters: Low energy $(<500 \mathrm{eV})$ particle measurements, Adv. Space Res., 1, 129-134, 1981.

Zesta, E., W. J. Hughes, M. J. Engebretson, T. J. Hughes, A. J. Lazarus, and K. I. Paularena, The November 9, 1993, traveling convection vortex event: A case study, J. Geophys. Res., 104, 28,041-28,058, 1999.

Zesta, E., W. J. Hughes, and M. J. Engebretson, A statistical study of traveling convection vortices using the magnetometer array for cusp and cleft studies, J. Geophys. Res., 107, 1317, doi:10.1029/1999JA000386, 2002.

R. B. Decker, Johns Hopkins University/APL, Laurel, MD 20723, USA.

D. G. Sibeck and A. Szabo, NASA/Goddard Space Flight Center, Code 696,8800 Greenbelt Road, Greenbelt, MD 20771, USA.(david.sibeck@) gsfc.nasa.gov)

H. J. Singer, NOAA Space Environment Center, Boulder, CO 80305, USA.

H. Tachihara, Earth and Planetary Sciences, University of Kyushu, Fukuoka 812-0053, Japan.

N. B. Trivedi, Laboratório de Ciências Espaciais de Santa Maria, Santa Maria, RS 97105-900, Brazil.

J. Watermann, Danish Meteorological Institute, Copenhagen DK-2100, Denmark.

E. Zesta, Atmospheric Sciences, University of California, Los Angeles, Los Angeles, CA 90095, USA. 